\section{SUBMARINE DISTURBANCE}

T $\mathrm{HE}$ following is an extract from the Meteorological log kept by Capt. R. J. Balderston on board the ship Belfast :-

"On December 22, I 884, at about ten minutes to 3 a.m., local ship's time, or $2 \mathrm{Id}$. I9h. $6 \mathrm{~m}$. Greenwich mean time, the ship Belfast, of Liverpool, was shaken by an earthquake which lasted from about 75 to 90 seconds. The vessel at the time was in latitude $34^{\circ} 34^{\prime}$ north and longitude $19^{\circ} 19^{\prime}$ west, the island of Madeira bearing true S.E., distant 145 miles.

"The shaking of the ship was accompanied by a loud rumbling noise, which, as heard from the cabin, resembled the sound which would be made by the rolling of large, empty, iron tanks about the decks, but which, as heard from the upper deck and in the open air, was as that of not very distant thunder, and it appeared to fill the whole of the air.

"I did not hear the commencement of the thunderous sound, and cannot say on what compass-bearing of the visible sky it commenced, but it travelled rapidly through the air and towards the S.W.

"The vibration of the vessel and the noise were greatest during the first 50 or 60 seconds ; the former then died gradually away and ended in the very faintest tremor, while the latter, as it travelled south-westward through the atmosphere, died out with a low roar as it appeared to sink beyond the horizon.

"The helmsman found the steering. wheel much shaken as he held it, and in the cabins and cook-house, tin ware, crockery ware, and other light articles were rattled about.

"This little earthquake occurred three days prior to the commencement of the earthquake which caused so much loss of life and property in Spain.

"Meteorological Office, October 9"

\section{THE BOTANICAL GARDENS IN JAVA}

$\mathrm{D}^{2}$

URING the last few years so many useful and important improvements have been made in the botanical gardens at Buitenzorg and Tsi-Bodas that it might not be amiss if the attention of the readers of NATURE were again drawn to these valuable seats of systematic and philosophical research.

On entering the gardens at Buitenzorg the stranger is at once struck with the wealth and luxuriance of the vegetation he sees, the great height of the trees whose trunks and branches are in many cases covered with heavy creepers, the dense copses of the different species of bamboo, the eccentric-looking screw-pines and the handsome palm trees; but the scientific observer is also struck with the care that has been taken to arrange all these many varieties of tropical plant life in, as far as possible, their systematic order, and that each specimen has its scientific, and in many cases its Malay name also, clearly and distinctly printed on a little board by its side.

It is not difficult for any one to find his way about the garden, and in a very short time he can discover the particular family or group of plants which he may desire to study. Many families have probably more representatives in these gardens than in any in the world. The Sapataceæ, for instance, so rarely seen in Europe, are here represented by a great variety of genera and species, and the Palmaceæ, the Rubiaceæ, the Burseraceæ, the Orchidaceæ, and other families have now a large number of rare and interesting representatives.

The herbarium which is attached to the garden contains a large collection of dried plants and seeds collected together from the many expeditions into the little or unknown parts of the archipelago and from other sources. Attached to the herbarium there is a comfortable and convenient little library which contains most of the mportant botanical books and journals.
The laboratory, which, thanks to the energy of Dr. Treub, the director, is now completed, is a large, lofty and, for these climes, particularly cool room, and is well fitted out with reagents and apparatus for carrying on botanical research. The generous invitation which Dr. Treub has issued to naturalists and to which the attention of the readers of NATURE has already been directed has attracted several scientific men of different nationalities, and some excellent research has already been made in this laboratory.

When I arrived in Buitenzorg Dr. Treub was at TsiBodas; so, after spending a few days in study in the gardens, I made the journey across the mountains to pay him a visit. The road from Buitenzorg to Tsi-Bodas crosses the Poenchuk Pass and is full of interest and beauty. On the way the traveller passes quite close to th: Talaga Werner, the crater of an extinct volcano which is now filled with water, and forms a most beautiful little lake hidden in the dense foliage of the mountain slopes. The path from the road to the lake is through a dense wood of fine forest trees, and amongst the undergrowth is found many fine shrubs and plants which are not found in the low-lying country beneath.

The gardens at Tsi-Bodas are situated on the slopes of the Gedeh Mountains, at an altitude of 5000 feet, and here $I$ found Dr. Treub at work in the comfortable little house which is attached to the gardens.

From this spot a very wide range of vegetation may be studied, from the rich and varied vegetation of the plains to the interesting vegetation of the Gedeh and Pangeranso peaks, at an elevation of Io,ooo feet. In the gardens themselves a very fine collection of Coniferæ from America, China, Australia, and other parts of the world has been got together, and spaces have been cleared for the growth of the various species of Eucalyptus, Cinchona, and other plants. Year by year the surrounding forest is being encroached upon by these gardens to make room for new importations. I was extremely sorry that I could not prolong my stay at Tsi Bodas, but I had to return to Batavia to catch the Molucca boat. I saw, however, enough to convince me of the great importance of these gardens for the advancement of our botanical knowledge and the great opportunities they afford for research into all branches of the science.

I need hardly say that the climate in this region is extremely pleasant and invigorating, and the neighbouring village of Sindanlaya is much resorted to by Europeans and others whose health has suffered on the coasts or low-lying districts of the Archipelago. At Buitenzorg the climate is by no means unpleasant or unhealthy, but as it lies a few thousand feet lower than Tsi-Bodas, it is naturally a good deal warmer; but I am assured that several Europeans have worked there for several years without feeling their health the least bit affected.

It is hardly necessary to add that every one who has come over to Java to work in these gardens has been amply repaid for the time spent in the long journey over the sea, for the insight which can be gained here into what tropical botany really is is one which can be gained nowhere else in the world so well, and leaves an impression which is not likely to be forgotten in a lifetime.

Batavia, July

SYDNEY J. HICKSON

ON CERTAIN NEW TERMS OR TERMS USED IN A NEW OR UNUSUAL SENSE IN ELEMENTARY UNIVERSAL GEOMETRY.

$$
\text { Point, Line, Plane, Space, Extension }
$$

A LINE may as usual be understood to mean a right line unless the contrary is stated.

Representable extension will comprise the concepts corresponding to the first four terms above written. So 
understood, the term a space is susceptible of a more precise meaning than is usually attributed to it: its intrinsic equation is given by Cayley's theorem of squared distances. It is a homaloid or flat of the $3 \mathrm{rd}$ as a plane is such of the 2 nd, a line of the Ist, and a point of the zeroth order.

The phrase space of the 4 th order ought accordingly to be superseded if we would avoid using the same word in two different senses-i.e. in a wider and narrower sense. Extension of the $4^{\text {th }}$ order is the proper expression to take its place, and so in general we ought to speak of extension of any given order $n$, and drop the phrase space of $n$-dimensions.

\section{Figure, Plasm, Enclosure}

A figure may exist in extension of any order. When pervasively limited by homaloids, simple and closed, I had proposed to give to it the provisional name of plasm, but Dr. Ingleby has supplied me with the more appropriate, or at least more simple, term, enclosure.

On the number and nature of simple regular enclosures in extension of any order, consult a remarkable memoir by Prof. Stringham* of the University of California (formerly of the Johns Hopkins University), in the third volume of the American Fournal of Mathematics.

Homaloid, Flat, Niveau, Absolute Measure of Distance

Homaloid, the term long ago introduced by the writer of this note, flat, suggested by the late lamented Clifford, are now well understood, and need no new explanation; but it is well to bear in mind the intrinsic equation which serves to define them to wit

A homaloid in extension of the nth order is definable by means of an equation of the second order (naturally expressible in the language of determinants), in which $(n+1)$ points are the standards of reference, and the squared distances from these of any other point in the homaloid are the coordinates.

Observe that the squared length is the absolute measure of distance between two points. The distances of each from the other are not equal but opposite quantities differing in algebraical sign.

A niveau is a very convenient term to signify the homaloid of the lowest order that can be drawn through a given point-group and is always unique; the order of the homaloid which is the niveau to a group of $n$ points cannot exceed $n-\mathrm{I}$.

Curves, surfaces, \&c., of the Ist, 2nd, and $n$th kind.

A plane (or simple) curve is of the first kind ; "a twisted curve," "courbe gauche," or a curve in extension of the 3 rd order, of the second kind, and in general a curve in extension of the $n$th order is a curve of the $(n-1)$ th kind.

Similarly we may define a simple surface as one of the first kind, and a surface in extension of the $n$th order as one of the $(n-2)$ th kind; and so in general a figure of variety $i+$ ( $i$ being I for a curve, 2 for a surface), in extension of the order $n$, is one of the $(n-i)$ th kind.

* Mr. Stringham, a native of "the bloody land" of Kansas, studied mathematics and fine art under Peirce and Norton, at Harvard, obtained a fellowship at the Johns Hopkins University, and completed his studies under Klein in Leipsic. In his memoir he has given perspective drawings of the bounding solids about a vertex of the regular figures in quaternary extension, such solids being supposed to be previously rotated round the vertex into the same space, which of course may be done just a; the bounding planes about a vertex of a regular figure in ternary extension may be rotated round that point into the same plane.

+ A curve may be called a one-dimensional, a surface a two-dimensional, a solid a three-dimensional continuum and, and so on. Thus a solid is to a space what a surface is to a plane and a curve to a right line.

t The ordinary systems of geometry, whether Euclidian or Non-Euclidian The ordinary systems of geometry, whether Euclidian or Non-Euclidian (Ultra-Euclidian would be the more correct term), contemplate figures as contained in homaloids of some order or another, but this limitation has an form for intonce pure theory of form ; for instance, a curve, i.e. a unidintensional continuum, may, and in general will, be such as cannot be contained in a homaloid of any number of case is infinite; but this would be a mere verbal quibble-the right view

\section{Curve, Locus, Assembly, Envelop, Environment}

A curve is that which is common to a locus of points and an assemblage of tangents; the locus is the envelop of the assembly, and the assemblage the environment of the locus.

\section{Lines and Points}

A line may be used in the double sense of a locus or direction. In the latter signification an Euclidian or objective line is the union of two lines running in contrary directions and an analytical line is a half-line, a " semidroite," meaning, of course, a half-Euclidian line.

So a point may mean either a position or an infinite assembly of lines (containing or) contained in it ; used in the latter sense, it might temporarily be termed a pencilpoint.

There are half or split points, as there are half or split lines. Thus the infinite extremities of the asymptotes to a hyperbola are half-points, the union of two of them being the correspondent to a single point in any ellipse of which the hyperbola is a perspective image.

\section{Coordinates, Homogeneous and Correlated}

Homogeneous systems of coordinates may be distinguished into absolute and proportional.

In the former the absolute magnitudes of each are material, in the latter their ratios only.

Also into direct and inverse.

Direct coordinates are measured by given multiples of the distances of a variable point from fixed homaloids; inverse by given multiples of the distances of a variable line, plane, \&c., from fixed points.

Correlated systems of direct and inverse coordinates are those in which my "universal mixed concomitant" (Clebsch's connex) $\xi x+\eta y+\zeta z$ (for greater clearness I confine myself for the moment to a particular diagrammatic case) equalled to zero expresses a line whose inverse coordinates are $\xi, \eta, \zeta$, when these are made constant and a point (pencil-point) whose direct coordinates (when it is regarded as denoting position) are $x, y, z$ when these in their turn are made constant.

If the distances of a point from the sides of the triangle of reference are $l, m, n$, and of a line from the angles of the same triangle $\lambda, \mu, \nu$, and if the direct coordinates being $c l, d m, e n$, and the inverse ones $\gamma \lambda, \delta \mu, \epsilon \nu$, and the distances of the angles from the sides $\not p, q, r-$

$$
c \gamma p=d \delta q=e \epsilon r \text {. }
$$

$l, m, n ; \lambda, \mu, \nu$ are correlated systems.

If $l^{\prime} m^{\prime} n^{\prime} p^{\prime} ; l, m, n, p$ the direct coordinates of two corresponding points in a homography are connected by the Matrix $M$ and $\lambda^{\prime} \mu^{\prime} \nu^{\prime} \pi^{\prime} ; \lambda, \mu, \nu, \pi$ (the inverse coordinates of two corresponding planes of the same homography) by the Matrix $M^{\prime}$, then if the two systems of coordinates are correlated, $M$ and $M^{\prime}$ will be opposite matrices.*

Of course the like will be true in extension of all orders: thus $e x . g r$. in the case of a plane if for a given homography

$$
\begin{aligned}
l^{\prime} & : a l+b m+c n \\
:: m^{\prime}: & d l+e m+f n \\
:: n^{\prime} & : g l+h m+k n
\end{aligned}
$$

Then

$$
\begin{array}{r}
\lambda^{\prime}:(e k-f h) \lambda+(f g-d k) \mu+(d h-e g) \nu \\
:: \mu^{\prime}:(c h-b k) \lambda+(a k-c g) \mu+(b g-a h) \nu \\
:: \nu^{\prime}:(b f-c e) \lambda+(e d-a f) \mu+(a e-b d) \nu
\end{array}
$$

being that it is sans niveau. The radical distinction therefore is not between the common Euclidian geometry and its generalisation (the socalled Non-Euclidian) but between the Homaloidal and the Anhomaloidal geometries.

words, fir two point-line, point-volume, \&c., schemes homoraphically related, employing correlated systems of proportional coordinates the matrix which serves to express the relation between the direct cordinates of the first coordinates of the first scheme and those of the second may be taken the transverse of the matrix which does the same between the inverse coordinates aware a new theorem. 
provided that $l, m, n ; \lambda, \mu, \nu$ are correlated systems of coordinates.

\section{Images : Reciprocals or Polar Reciprocals}

It is very convenient to speak of any function which equated to zero expresses a figure as an image* of such figure; thus ex. $g r . \xi x+\eta y+\xi z$ may be spoken of as an image of the line $\xi, \eta, \zeta$ and of the point $x, y, z$.

A curve being the concept common to a locus and an assembly (the common ground, so to say, of the existence of each of them), will be capable of being imaged in terms of either direct or inverse coordinates. If the two coordinate systems are supposed to be correlated (as they ought always to be) then any two homogeneous functions which are reciprocal, or, let us say, conjugate to one another (each in common parlance the polar reciprocal of the other) will be images-the one of the curve under its aspect as a locus, the other of the very same curve under its aspect as an assemblage.

\section{Reduced Perpendicular Distances}

An extremely convenient system of homogeneous coordinates of a point is where each coordinate is the distance from one of the boundaries of the fundamental enclosure divided by the distance of that boundary from the opposite angle. Such coordinates may be termed coordinates of reduced distance or reduced coordinates ; they are analytically defined by their sum being unity. If $a, b$ be the two vertices which correspond to the coordinates of reduced distances, the squared distance of any two points, $x, y, z, \ldots ; x^{\prime}, y^{\prime} z^{\prime}, \ldots$ in extension of any order is capable of being expressed by the formula $\Sigma(a b)^{2}\left(x-x^{\prime}\right)\left(y^{\prime}-y\right)$, which, as far as I have been able to ascertain, is nowhere stated in the books, except for the case of trilinear coordinates.

\section{Exihangeable Figures}

Two figures indistinguishable from each other by any of their internal properties, but incapable of occupying the same place (such as the left-and right-hand glove or shoe) have received the very awkward and misleading name of symmetrical figures; I propose to call them exchangeable figures, inasmuch as in the nature of things, as they are in themselves (without regard to the limitation of the human faculties), they may be made to pass into each other's places by a semi-revolution about a suitable homaloidal axis.

The Point-Pair at Infinity, Lines and Planes of Null

It has been already shown in these columns that the "absolute" in a plane has full right to be called the point-pair at infinity, in analogy with the received expression of the line at infinity, and those who have considered what has been here stated under the head of reciprocity will see good grounds for admitting that the line at infinity ought to be regarded as a complete line, i.e. as made up of two analytical "semi-droites."

Every line through either half of the absolute besides the property of being infinitely distant from any point in the finite region may be termed a line of mull, in the sense that the distance between any two points in such line is zero.

In like manner any plane touching the absolute in ex tension of the 3 rd order, besides being infinitely distant from the finite region, is in the same sense a plane of null; in it, form is divorced from content, for a figure of any shape being described upon such plane, its content will be nil.

\section{Pluri-duality: Containing and Contained}

In extension of $i$ dimensions each continuum of $\lambda$ dimensions stands in a relation of reciprocity to one of

* When an image is given, its object is absolutely determined, but not vace vers $\hat{a}$, since an image may te magnified or diminished at will by the introduction of a constant factor.
$i-\lambda-\mathrm{I}$ dimensions, the total number of these "dualities" being $\frac{i+1}{2}$ when $i$ is odd and $\frac{i}{2}$ when $i$ is even (in the former case the continuum of $\frac{i-1}{2}$ dimensions being its own reciprocal). It is very convenient in connecting reciprocal geometrical statements to ignore the difference between (and to regard as exchangeable and equivalent) the terms contoining and contained in as applied to heterogeneous continua; indeed the ordinary distinctive use of these words suggests an erroneous conception; as ex. gr. of a line being made $u p$ of points or a plane of lines. A point may be said to contain every line or plane which passes through it, and a line every point which lies on it, and every plane which passes through it: as an example of this extended locution the order, rank, and class of a surface may be defined as follows-viz. the order and class as the number of its point and plane elements respectively contained in any given line; the rank as the number of its line elements contained in common by any given point and plane which contain one another.

A plane-section of a surface is the totality of its pointor line-elements contained in a plane and similarly a point-section (an enveloping cone), the totality of its plane- or line-elements contained in a point : hence indifferently the class of any plane-section or the order of any point-section of a surface is its rank.*

\section{J. J. SylVESTER}

\section{NOTES}

ALL the five French academies will celebrate by a banquet the ninetieth anniversary of the foundation of the Institut, which was established on October 25, 1795, by the Conseil Législatif and Directoire Executif of the French Republic. The actual organisation is not quite the same as the original, great alterations having been made in 1814 , and only partially abolished on subsequent occasions.

THE death took place last month of General J. J. Baeyer, President of the Central Bureau for European Triangulation and of the Royal Prussian Geodetic Institute. General Baeyer had reached the age of ninety-one years. A biography of some length will be found in the Astronomische Nachrichten, No. 2687.

M. Robin, a member of the Paris Academy of Sciences and of the French Senate, died last week. He had devoted his exertions to microscopy, and was professor to the School of Medicine.

* The word spread, to signify an unimited expanse of discontinuous points and so used by Dr. Henrici, is, I am informed, originally due to the late Prof. Clifford. In ignurance of this fact, on hearing that Henrici had been attacked for his use of the word, I stated my belief that it must have been borrowed from my use of it to signify a limited portion of a tissue of equi-spaced points, such as that which is turned to so profitable account in my constructive theory of partitions in the American Fournal of Mathematics. I did not know at the time that Clifford had used the word, nor that Dr. Henrici's treatise preceded by several years the publication of my memoir above referred to. This erroneous oral statement seems to have found its way by some more or less circuitous channel to the culumns of the Saturday Review in a notice of a criticism, by Mr. Dodsson, of Dr. Henrici's geoReziew in a notice of a criticism, by Mr. Dodgson, of Dr. Henrici's geo-
metrical manual in the Scientific Series. Dr. Ferrers (the Master of Caius metrical manual in the Scientific Series. Dr. Ferrers (the Master of Caius
College, Cambridge) was the first to apply a spread to demonstrate in College, Cambridge) was the first to apply a spread to demonstrate in tuitively a celebrated arithmetical theorem of reciprocity due to Euler. Nr. Durfee a quarter of a century later led the way to a further and more pregnant use of the same by showing how to trisect a symmetrical spread bounded by two right lines and a broken line into a regular square and two quasi-triangular appendages, to which I superadded the notion of mult.secting it into a succession of angles. Another pupil of mine at the Johns Hopkins University (Mr. Ely) has laid the foundation of a new theory of partitions, by studying the various modes of decomposing a solid
spread of discontinuous points; his memoir on the subject is to be found in a recent volume of the A merican Mathematical Fournal. By means of the trisection method I obtained inter alia a new expansion
of $(\mathrm{I}-x z)\left(\mathrm{I}-x^{2} z\right) \ldots\left(\mathrm{I}-x^{2} z\right)$, which, on making $z$ unity and $n$ infinite of $(\mathrm{I}-x \%)\left(\mathrm{I}-x^{2} z\right) \ldots\left(\mathrm{I}-x^{2} z\right)$, which, on making $z$ unity and $n$ infinite results of a totally novel kind by the multisection methed: so that a spread may justly be regarded as a potent instrument or magical mirror for extending old and bringing to view new truths in the alonderland of partition and elliptic-function series. 